Supplementary Materials for

\title{
PAT Implementation on a Mobile Continuous Pharmaceutical Manufacturing System: Real-Time Process Monitoring with In-line FTIR and Raman Spectroscopy
}

Yuma Miyai ${ }^{a s}$, Anna Formosa ${ }^{b s}$, Cameron Armstronga, Brian Marquardtc, Luke Rogers ${ }^{b}$,

\section{Thomas Roper ${ }^{a^{*}}$}

a Virginia Commonwealth University, Department of Chemical and Life Science Engineering Richmond, Virginia 23284-2512, United States

${ }^{\mathrm{b}}$ OnDemand Pharmaceuticals, Rockville, Maryland 20850, United States

${ }^{c}$ MarqMetrix Inc., Seattle, Washington 98103, United States

*Email: tdroper@vcu.edu

$\S Y . M$. and A.F. contributed equally to this paper

This PDF file includes:

Synthesis Parameters

Conditions for Heat Transfer Profile with Computational Fluid Dynamics (CFD)

HPLC Analytical Method

PAT Data Collection and Management

Supplemental Tables S1-S8

Supplemental Figures S1-S8 


\section{Synthesis Parameters}

The optimized five-step continuous ciprofloxacin synthesis method was developed in the previous publication. ${ }^{1}$ In this process, the first three steps are telescoped and the material is collected. This material is carried on to the last two steps which are telescoped together for the crude API synthesis. The solutions for $1.0 \mathrm{M}$ acyl chloride 1, 1.2 M acrylate 2 with 1.37 M DIPEA, and 1.25 M cyclopropylamine 4 were prepared with ACS grade acetonitrile. Step 1 consists of acyl chloride 1 and acrylate 2 solutions each flowing at $2.5 \mathrm{~mL} / \mathrm{min}$ at $150{ }^{\circ} \mathrm{C}$ with 2 minutes residence time. The reaction stream exiting Step 1 (approximately $150 \mathrm{mg} / \mathrm{mL}$ of keto-ester 3) meets with cyclopropylamine 4 solution flowing at $2.5 \mathrm{~mL} / \mathrm{min}$ and enters reaction step 2 at room temperature with 40 seconds residence time. $12.5 \mathrm{~mL} / \mathrm{min} 1 \mathrm{M} \mathrm{HCl}$ and $5 \mathrm{~mL} / \mathrm{min} 2-\mathrm{MeTHF}$ streams are mixed with the stream exiting Step 2 (approximately $95 \mathrm{mg} / \mathrm{mL}$ of cyclopropyl-enamine 5) and the combined stream enters a gravity separator where cyclopropyl-enamine $\mathbf{5}$ is extracted from acetonitrile and into 2-MeTHF at approximately $130 \mathrm{mg} / \mathrm{mL}$. Step 3 material is collected in a vessel and fed into Step 4 at $4 \mathrm{ml} / \mathrm{min}$ along with a mixture of DMSO, piperazine $(0.735 \mathrm{M})$, and TBAOH solution $(0.315 \mathrm{M})$ at $8 \mathrm{~mL} / \mathrm{min}$ where Step 4 is operated at $150{ }^{\circ} \mathrm{C}$ with the residence time of 5 minutes. The outlet stream of Step 4 is combined with $1.5 \mathrm{M}$ TBAOH solution $(2.2$ $\mathrm{mL} / \mathrm{min}$ ) and the stream enters Step 5 where the reaction takes place at $150{ }^{\circ} \mathrm{C}$ with a residence time of 3.2 minutes. Two BPRs were set to 175 psi each and were placed after Step 2 and Step 5.

\section{Heat Transfer Profiles and CFD}

The lengths of tubing tested for maintaining a high temperature into the Raman probe were modeled using Comsol Multiphysics version-5.5 (Burlington, MA). A 2D-axisymmetric geometry was applied to capture the phenomena in a computationally efficient manner, and the temperature profiles of each tubing length were solved for assuming laminar flow and constant physical parameters of the primary solvent, dimethyl sulfoxide (DMSO).

\section{Liquid Chromatography (LC) Parameters}

The HPLC analytical method used for this work was reported in the previous publication. ${ }^{1}$ An Agilent 1200 LC-VWD with a Phenomenex Luna $5 \mu \mathrm{m} \mathrm{C18} 250$ x $4.6 \mathrm{~mm}$ column was used for collecting all LC data. A $25 \mathrm{mM}$ phosphate buffer $(\mathrm{pH}=3 \pm 0.05)$ was prepared by adding phosphoric acid to water and adjusting the $\mathrm{pH}$ to 3 with triethylamine. The buffer was used for aqueous mobile phase (mobile phase A) and methanol was used for the organic mobile phase (mobile phase B). The LC method for this study includes $5 \mu \mathrm{L}$ injection volume and $55{ }^{\circ} \mathrm{C}$ oven temperature collected at $278 \mathrm{~nm}$. Step 2 samples were prepared in diluent 2 comprised of $80 \%$ mobile phase A and $20 \%$ acetonitrile (HPLC grade). Step 5 samples were prepared in diluent 1 
comprised of $20 \%$ mobile phase A and $80 \%$ acetonitrile (HPLC grade). Step 2 samples were diluted 166x and Step 5 samples were diluted 200x prior to injection.

Typical chromatograms for cyclopropyl-enamine $\mathbf{5}$ and ciprofloxacin $\mathbf{8}$ standards used for building concentration models are shown in Figures S1 and S3 and Tables S1 and S3. Steps 2 and 5 crude reaction mixtures operated in flow at the normal operating condition are shown in Figures S2 and S4 and Tables S2 and S4.

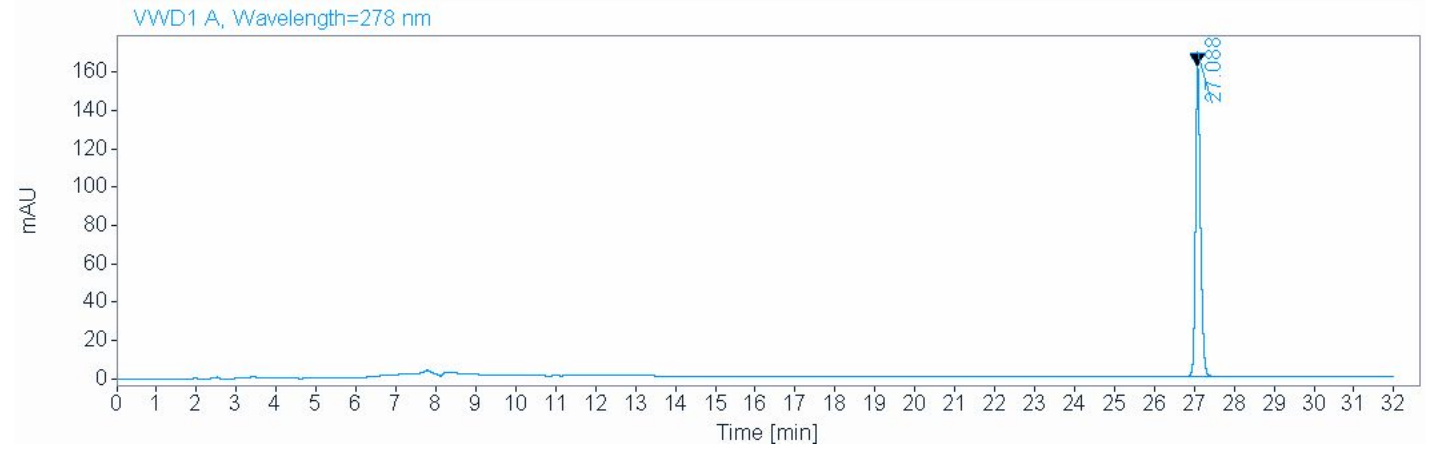

Figure S1. Chromatogram of cyclopropyl-enamine 5 standard

Table S1. Integration of cyclopropyl-enamine $\mathbf{5}$ standard Peak Retention Time Area Peak Area Percent Height

$\begin{array}{llll}27.088 & 1411.00 & 100.00 & 161.90\end{array}$

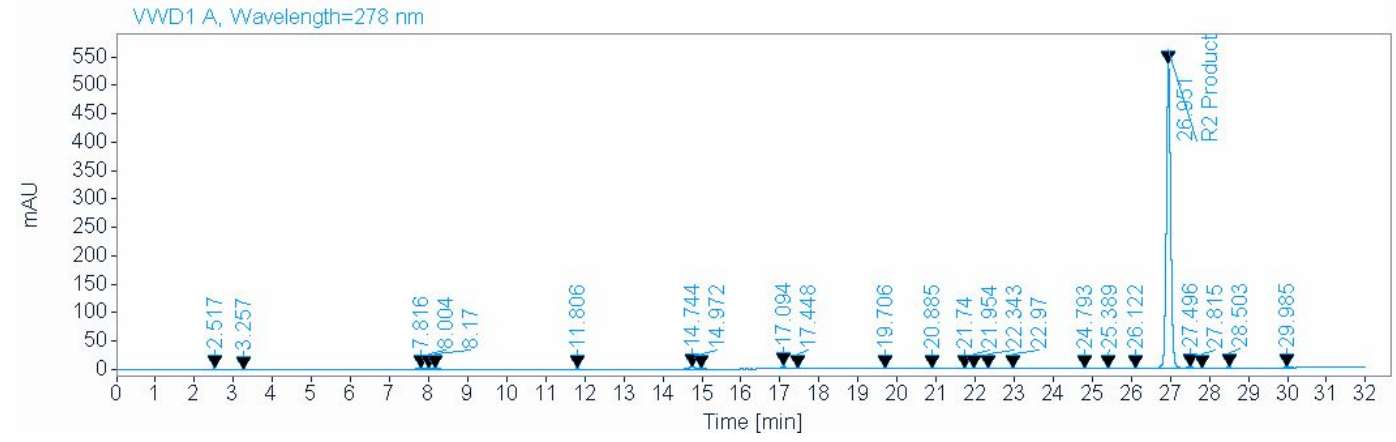

Figure S2. Chromatogram of Step 2 crude reaction sample 
Table S2. Integration of Step 2 crude reaction sample

\begin{tabular}{cccc} 
Peak Retention Time & Area & Peak Area Percent & Height \\
\hline 2.517 & 4.08 & 0.09 & 0.77 \\
3.257 & 2.85 & 0.07 & 0.19 \\
7.816 & 27.75 & 0.63 & 1.57 \\
8.004 & 19.49 & 0.44 & 2.04 \\
8.17 & 22.07 & 0.50 & 2.47 \\
11.806 & 2.40 & 0.05 & 0.28 \\
14.744 & 43.76 & 1.00 & 4.64 \\
14.972 & 9.67 & 0.22 & 1.25 \\
17.094 & 41.83 & 0.95 & 4.87 \\
17.448 & 5.11 & 0.12 & 0.34 \\
19.706 & 2.41 & 0.05 & 0.21 \\
20.885 & 6.51 & 0.15 & 0.63 \\
21.74 & 2.82 & 0.06 & 0.36 \\
21.954 & 5.60 & 0.13 & 0.56 \\
22.343 & 5.48 & 0.12 & 0.81 \\
22.97 & 11.50 & 0.26 & 1.33 \\
24.793 & 3.93 & 0.09 & 0.55 \\
25.389 & 5.68 & 0.13 & 0.48 \\
26.122 & 2.37 & 0.05 & 0.30 \\
26.951 & 4120.40 & 93.90 & 535.60 \\
27.496 & 26.37 & 0.60 & 2.34 \\
27.815 & 2.54 & 0.06 & 0.33 \\
28.503 & 8.44 & 0.19 & 1.10 \\
29.985 & 4.96 & 0.11 & 0.62
\end{tabular}

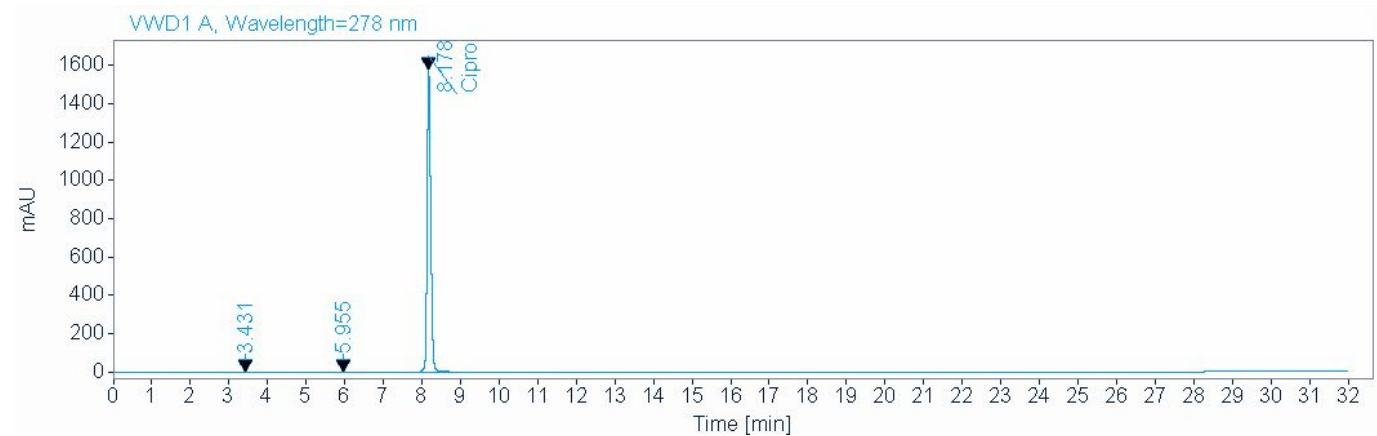

Figure S3. Chromatogram of ciprofloxacin 8 product standard

Table S3. Integration of ciprofloxacin $\mathbf{8}$ standard

\begin{tabular}{|c|c|c|c|}
\hline Peak Retention Time & Area & PeakArea Percent & Height \\
\hline 3.431 & 3.19 & 0.03 & 0.65 \\
\hline 5.955 & 6.77 & 0.07 & 1.13 \\
\hline 8.178 & 10272.00 & 99.90 & 1577.00 \\
\hline
\end{tabular}




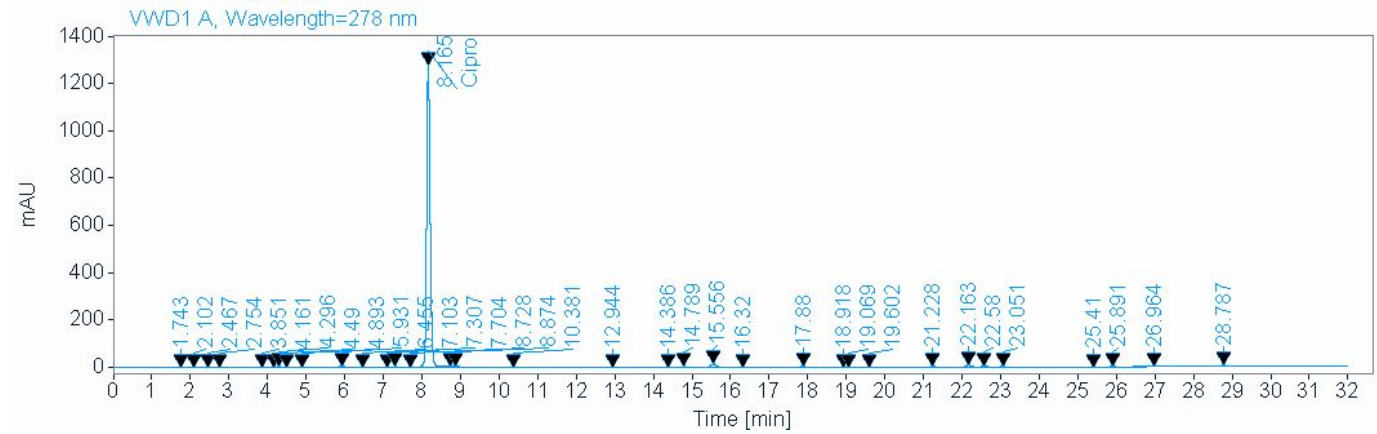

Figure S4. Chromatogram Step 5 crude reaction sample

Table S4. Integration of Step 5 crude reaction sample

\begin{tabular}{|c|c|c|c|}
\hline Peak Retention Time & Area & PeakArea Percent & Height \\
\hline 1.743 & 3.77 & 0.04 & 0.74 \\
\hline 2.102 & 5.76 & 0.07 & 0.77 \\
\hline 2.467 & 3.65 & 0.04 & 0.69 \\
\hline 2.754 & 3.69 & 0.04 & 0.26 \\
\hline 3.851 & 3.15 & 0.04 & 0.29 \\
\hline 4.161 & 8.31 & 0.10 & 1.34 \\
\hline 4.296 & 14.20 & 0.16 & 2.50 \\
\hline 4.49 & 4.06 & 0.05 & 0.82 \\
\hline 4.893 & 2.43 & 0.03 & 0.45 \\
\hline 5.931 & 40.05 & 0.46 & 6.70 \\
\hline 6.455 & 5.91 & 0.07 & 0.61 \\
\hline 7.103 & 7.42 & 0.09 & 1.28 \\
\hline 7.307 & 9.63 & 0.11 & 1.57 \\
\hline 7.704 & 2.52 & 0.03 & 0.46 \\
\hline 8.165 & 8171.30 & 94.40 & 1279.00 \\
\hline 8.728 & 2.16 & 0.02 & 0.40 \\
\hline 8.874 & 16.74 & 0.19 & 2.29 \\
\hline 10.381 & 3.48 & 0.04 & 0.53 \\
\hline 12.944 & 4.71 & 0.05 & 0.51 \\
\hline 14.386 & 4.64 & 0.05 & 0.38 \\
\hline 14.789 & 11.08 & 0.13 & 1.25 \\
\hline 15.556 & 105.49 & 1.22 & 13.02 \\
\hline 16.32 & 5.45 & 0.06 & 0.69 \\
\hline 17.88 & 12.33 & 0.14 & 1.47 \\
\hline 18.918 & 6.23 & 0.07 & 0.75 \\
\hline 19.069 & 4.80 & 0.06 & 0.58 \\
\hline 19.602 & 2.38 & 0.03 & 0.17 \\
\hline 21.228 & 10.80 & 0.12 & 1.18 \\
\hline 22.163 & 62.10 & 0.72 & 7.67 \\
\hline 22.58 & 22.75 & 0.26 & 2.77 \\
\hline 23.051 & 6.82 & 0.08 & 0.84 \\
\hline 25.41 & 2.71 & 0.03 & 0.21 \\
\hline 25.891 & 2.34 & 0.03 & 0.19 \\
\hline 26.964 & 3.63 & 0.04 & 0.19 \\
\hline 28.787 & 79.13 & 0.91 & 5.81 \\
\hline
\end{tabular}


A

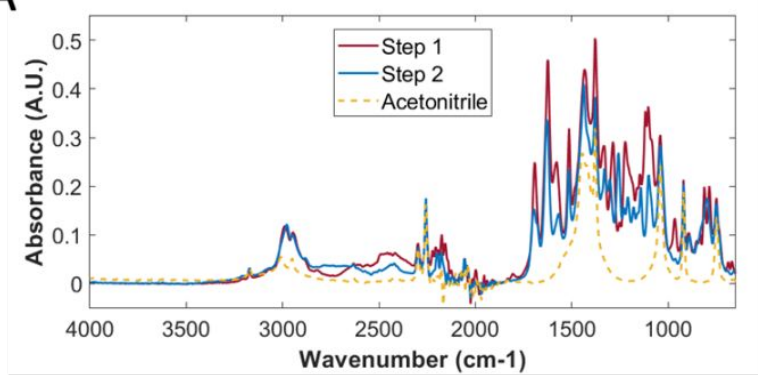

B

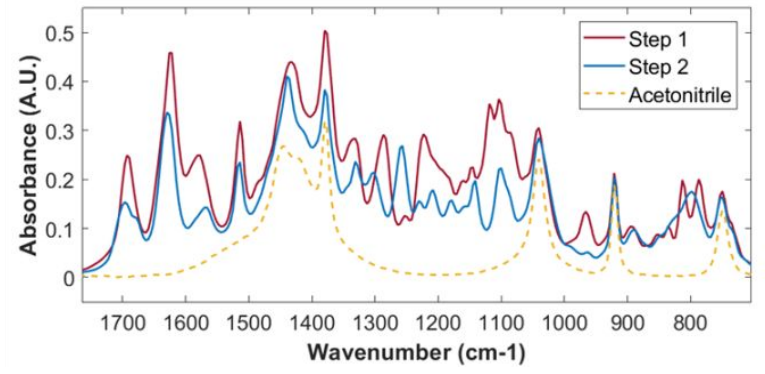

Figure S5. Raw spectral data of Step 2 FlowIR model: (A) IR spectra of acetonitrile and Step 1 and Step 2 crude solutions and (B) a spectra region from 704 to $1764 \mathrm{~cm}^{-1}$ was used for building Step 2 FlowIR model.

Table S5. A total of 97 calibration samples were used for building Step 2 FlowIR model (Figure 4C) for in-line monitoring of the cyclopropyl-enamine 5 concentrations in the Step 2 reaction stream. The actual concentration values were compared to the predicted values by the model for determining the relative error for each sample. 


\begin{tabular}{|c|c|c|c|c|c|c|c|}
\hline Sample & $\begin{array}{l}\text { Actual Concentration } \\
\qquad(\mathrm{mg} / \mathrm{mL})\end{array}$ & $\begin{array}{l}\text { Predicted Concentration } \\
\qquad(\mathrm{mg} / \mathrm{mL})\end{array}$ & $\begin{array}{c}\text { Relative Error } \\
\qquad(\%)\end{array}$ & Sample & $\begin{array}{l}\text { Actual Concentration } \\
\qquad(\mathrm{mg} / \mathrm{mL})\end{array}$ & $\begin{array}{l}\text { Predicted Concentration } \\
\qquad(\mathrm{mg} / \mathrm{mL})\end{array}$ & $\begin{array}{c}\text { Relative Error } \\
(\%)\end{array}$ \\
\hline 1 & 129.3 & 128.3 & $0.8 \%$ & 51 & 99.0 & 97.9 & $1.2 \%$ \\
\hline 2 & 118.7 & 120.2 & $1.3 \%$ & 52 & 99.8 & 97.9 & $1.8 \%$ \\
\hline 3 & 109.2 & 112.1 & $2.7 \%$ & 53 & 92.4 & 94.3 & $2.0 \%$ \\
\hline 4 & 99.3 & 103.7 & $4.4 \%$ & 54 & 95.6 & 94.3 & $1.3 \%$ \\
\hline 5 & 91.7 & 95.4 & $4.0 \%$ & 55 & 100.0 & 98.2 & $1.8 \%$ \\
\hline 6 & 86.6 & 91.0 & $5.1 \%$ & 56 & 99.5 & 98.2 & $1.3 \%$ \\
\hline 7 & 82.1 & 86.6 & $5.5 \%$ & 57 & 52.4 & 52.1 & $0.6 \%$ \\
\hline 8 & 73.9 & 78.1 & $5.6 \%$ & 58 & 54.4 & 52.0 & $4.3 \%$ \\
\hline 9 & 66.6 & 69.2 & $3.8 \%$ & 59 & 64.0 & 63.3 & $1.2 \%$ \\
\hline 10 & 56.6 & 60.1 & $6.1 \%$ & 60 & 95.9 & 95.8 & $0.1 \%$ \\
\hline 11 & 48.6 & 51.2 & $5.3 \%$ & 61 & 80.3 & 80.5 & $0.2 \%$ \\
\hline 12 & 134.1 & 126.7 & $5.5 \%$ & 62 & 90.2 & 89.9 & $0.4 \%$ \\
\hline 13 & 119.9 & 115.6 & $3.6 \%$ & 63 & 39.1 & 40.3 & $3.0 \%$ \\
\hline 14 & 111.3 & 109.4 & $1.7 \%$ & 64 & 38.2 & 38.9 & $1.9 \%$ \\
\hline 15 & 111.0 & 105.4 & $5.1 \%$ & 65 & 47.7 & 48.7 & $2.1 \%$ \\
\hline 16 & 108.9 & 101.3 & $7.0 \%$ & 66 & 57.1 & 58.2 & $1.9 \%$ \\
\hline 17 & 98.1 & 97.1 & $1.0 \%$ & 67 & 66.5 & 68.1 & $2.4 \%$ \\
\hline 18 & 98.0 & 93.0 & $5.1 \%$ & 68 & 76.0 & 77.6 & $2.1 \%$ \\
\hline 19 & 90.1 & 88.5 & $1.8 \%$ & 69 & 80.7 & 82.1 & $1.7 \%$ \\
\hline 20 & 83.7 & 83.8 & $0.1 \%$ & 70 & 85.6 & 87.2 & $1.9 \%$ \\
\hline 21 & 81.5 & 78.7 & $3.4 \%$ & 71 & 90.4 & 92.5 & $2.3 \%$ \\
\hline 22 & 73.3 & 74.2 & $1.2 \%$ & 72 & 95.0 & 97.3 & $2.4 \%$ \\
\hline 23 & 65.2 & 64.6 & $1.0 \%$ & 73 & 99.7 & 100.7 & $1.0 \%$ \\
\hline 24 & 56.7 & 55.0 & $3.0 \%$ & 74 & 104.5 & 105.9 & $1.4 \%$ \\
\hline 25 & 46.5 & 45.5 & $2.0 \%$ & 75 & 109.4 & 110.9 & $1.4 \%$ \\
\hline 26 & 37.4 & 35.8 & $4.3 \%$ & 76 & 114.0 & 115.4 & $1.2 \%$ \\
\hline 27 & 37.4 & 37.2 & $0.4 \%$ & 77 & 123.4 & 123.7 & $0.3 \%$ \\
\hline 28 & 56.2 & 50.0 & $11.0 \%$ & 78 & 133.2 & 133.5 & $0.3 \%$ \\
\hline 29 & 66.5 & 65.0 & $2.3 \%$ & 79 & 38.1 & 37.1 & $2.7 \%$ \\
\hline 30 & 83.8 & 74.7 & $10.8 \%$ & 80 & 42.8 & 42.2 & $1.6 \%$ \\
\hline 31 & 109.9 & 110.1 & $0.2 \%$ & 81 & 47.5 & 47.0 & $1.0 \%$ \\
\hline 32 & 124.4 & 125.7 & $1.0 \%$ & 82 & 52.2 & 51.8 & $0.6 \%$ \\
\hline 33 & 98.4 & 96.8 & $1.6 \%$ & 83 & 57.1 & 57.1 & $0.0 \%$ \\
\hline 34 & 100.5 & 104.1 & $3.6 \%$ & 84 & 61.9 & 61.5 & $0.6 \%$ \\
\hline 35 & 96.3 & 95.8 & $0.6 \%$ & 85 & 66.4 & 66.4 & $0.0 \%$ \\
\hline 36 & 72.0 & 74.6 & $3.7 \%$ & 86 & 71.3 & 71.2 & $0.1 \%$ \\
\hline 37 & 45.7 & 47.6 & $4.0 \%$ & 87 & 76.1 & 76.3 & $0.2 \%$ \\
\hline 38 & 101.7 & 103.0 & $1.2 \%$ & 88 & 80.7 & 80.6 & $0.0 \%$ \\
\hline 39 & 94.2 & 97.7 & $3.7 \%$ & 89 & 85.6 & 85.4 & $0.2 \%$ \\
\hline 40 & 91.3 & 96.2 & $5.4 \%$ & 90 & 90.2 & 89.9 & $0.3 \%$ \\
\hline 41 & 94.4 & 94.0 & $0.5 \%$ & 91 & 94.9 & 94.4 & $0.5 \%$ \\
\hline 42 & 97.6 & 94.2 & $3.5 \%$ & 92 & 99.9 & 99.0 & $0.9 \%$ \\
\hline 43 & 94.3 & 99.8 & $5.9 \%$ & 93 & 104.6 & 103.8 & $0.8 \%$ \\
\hline 44 & 93.5 & 100.8 & $7.8 \%$ & 94 & 109.3 & 108.7 & $0.6 \%$ \\
\hline 45 & 98.2 & 98.3 & $0.1 \%$ & 95 & 113.9 & 113.3 & $0.5 \%$ \\
\hline 46 & 97.9 & 98.5 & $0.6 \%$ & 96 & 123.4 & 121.9 & $1.3 \%$ \\
\hline 47 & 99.2 & 98.8 & $0.4 \%$ & 97 & 133.0 & 130.5 & $1.9 \%$ \\
\hline 48 & 96.4 & 98.7 & $2.4 \%$ & & & & \\
\hline 49 & 68.4 & 64.4 & $5.8 \%$ & & & & \\
\hline 50 & 66.5 & 64.4 & $3.1 \%$ & & & & \\
\hline
\end{tabular}


A

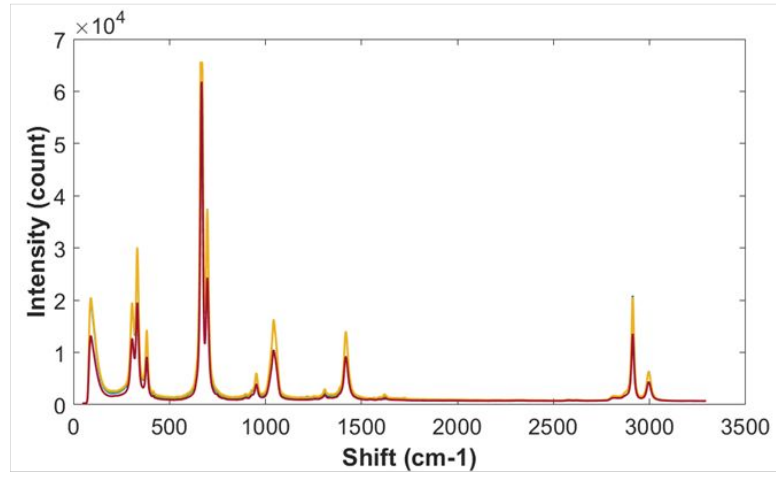

B

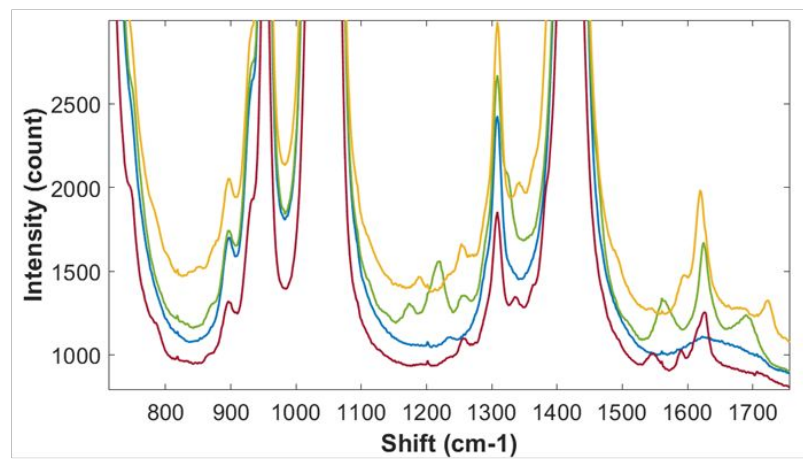

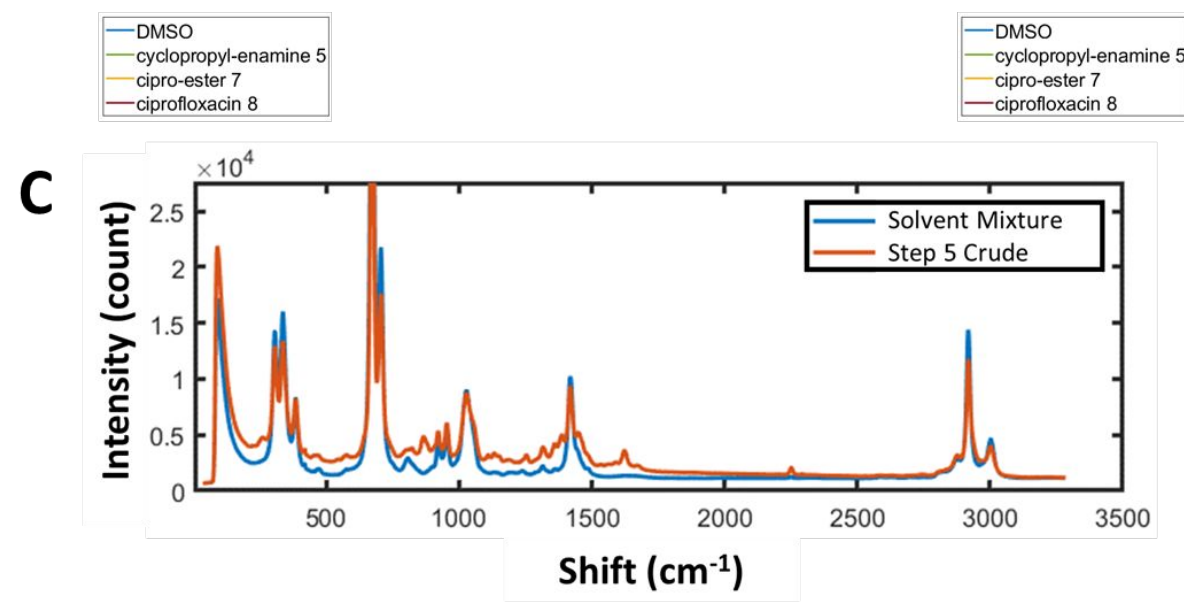

Figure S6. Spectral data of Step 5 Raman model: (A) Raman spectra of DMSO, cyclopropylenamine 5, cipro-ester 7, and ciprofloxacin 8, (B) spectra regions from 768 to $971 \mathrm{~cm}^{-1}$ and from 1077 to $1660 \mathrm{~cm}^{-1}$ were used for Step 5 Raman model and the peak intensity was increased to show small peaks which were important for the model, and (C) Raman spectra of Step 5 crude solution compared to the solvent mixture (water/2-MeTHF/DMSO) used for Step 4-5 reaction.

Table S6. A total of 165 calibration samples were used for building Step 5 Raman model (Figure 6C) for in-line monitoring of the ciprofloxacin 8 concentrations in the Step 5 reaction stream. The actual concentration values were compared to the predicted values by the model and the relative error for each sample was calculated. 


\begin{tabular}{|c|c|c|c|c|c|c|c|}
\hline Sample & $\begin{array}{l}\text { Actual Concentration } \\
\qquad(\mathrm{mg} / \mathrm{mL})\end{array}$ & $\begin{array}{l}\text { Predicted Concentration } \\
\qquad(\mathrm{mg} / \mathrm{mL})\end{array}$ & Relative Error (\%) & Sample & $\begin{array}{l}\text { Actual Concentration } \\
(\mathrm{mg} / \mathrm{mL})\end{array}$ & $\begin{array}{l}\text { Predicted Concentration } \\
\qquad(\mathrm{mg} / \mathrm{mL})\end{array}$ & Relative Error (\%) \\
\hline 1 & 17.84 & 17.6 & $1.5 \%$ & 51 & 27.2 & 26.9 & $1.1 \%$ \\
\hline 2 & 17.84 & 17.3 & $2.7 \%$ & 52 & 27.2 & 26.8 & $1.6 \%$ \\
\hline 3 & 17.84 & 17.6 & $1.4 \%$ & 53 & 27.2 & 26.8 & $1.4 \%$ \\
\hline 4 & 17.84 & 17.6 & $1.2 \%$ & 54 & 27.2 & 26.9 & $1.3 \%$ \\
\hline 5 & 17.84 & 17.4 & $2.4 \%$ & 55 & 27.2 & 27.0 & $0.8 \%$ \\
\hline 6 & 19.27 & 19.9 & $3.1 \%$ & 56 & 30.6 & 29.8 & $2.5 \%$ \\
\hline 7 & 19.27 & 18.7 & $2.8 \%$ & 57 & 30.6 & 29.9 & $2.4 \%$ \\
\hline 8 & 19.27 & 19.6 & $1.9 \%$ & 58 & 30.6 & 29.7 & $2.8 \%$ \\
\hline 9 & 19.27 & 19.5 & $1.2 \%$ & 59 & 30.6 & 29.7 & $2.8 \%$ \\
\hline 10 & 19.27 & 19.7 & $2.4 \%$ & 60 & 30.6 & 29.7 & $2.9 \%$ \\
\hline 11 & 27.83 & 28.5 & $2.3 \%$ & 61 & 32.3 & 31.4 & $2.7 \%$ \\
\hline 12 & 27.83 & 28.3 & $1.8 \%$ & 62 & 32.3 & 31.4 & $2.6 \%$ \\
\hline 13 & 27.83 & 28.5 & $2.5 \%$ & 63 & 32.3 & 31.5 & $2.4 \%$ \\
\hline 14 & 27.83 & 28.3 & $1.7 \%$ & 64 & 32.3 & 31.5 & $2.6 \%$ \\
\hline 15 & 27.83 & 28.5 & $2.3 \%$ & 65 & 32.3 & 31.5 & $2.4 \%$ \\
\hline 16 & 34.29 & 35.0 & $2.0 \%$ & 66 & 34.0 & 34.4 & $1.1 \%$ \\
\hline 17 & 34.29 & 34.8 & $1.6 \%$ & 67 & 34.0 & 34.5 & $1.4 \%$ \\
\hline 18 & 34.29 & 34.8 & $1.5 \%$ & 68 & 34.0 & 34.6 & $1.6 \%$ \\
\hline 19 & 34.29 & 34.8 & $1.5 \%$ & 69 & 34.0 & 34.3 & $0.9 \%$ \\
\hline 20 & 34.29 & 34.8 & $1.4 \%$ & 70 & 34.0 & 34.3 & $0.8 \%$ \\
\hline 21 & 38.07 & 38.0 & $0.1 \%$ & 71 & 35.7 & 35.4 & $0.8 \%$ \\
\hline 22 & 38.07 & 38.2 & $0.4 \%$ & 72 & 35.7 & 35.1 & $1.7 \%$ \\
\hline 23 & 38.07 & 38.0 & $0.1 \%$ & 73 & 35.7 & 35.2 & $1.4 \%$ \\
\hline 24 & 38.07 & 38.2 & $0.2 \%$ & 74 & 35.7 & 35.3 & $1.2 \%$ \\
\hline 25 & 38.07 & 38.1 & $0.1 \%$ & 75 & 35.7 & 35.2 & $1.3 \%$ \\
\hline 26 & 40.18 & 41.6 & $3.5 \%$ & 76 & 37.4 & 37.0 & $1.1 \%$ \\
\hline 27 & 40.18 & 41.4 & $2.9 \%$ & 77 & 37.4 & 36.9 & $1.3 \%$ \\
\hline 28 & 40.18 & 41.3 & $2.9 \%$ & 78 & 37.4 & 36.9 & $1.5 \%$ \\
\hline 29 & 40.18 & 41.1 & $2.4 \%$ & 79 & 37.4 & 37.1 & $0.9 \%$ \\
\hline 30 & 40.18 & 40.9 & $1.7 \%$ & 80 & 37.4 & 36.9 & $1.3 \%$ \\
\hline 31 & 40.74 & 41.1 & $1.0 \%$ & 81 & 39.5 & 39.2 & $0.7 \%$ \\
\hline 32 & 40.74 & 40.6 & $0.4 \%$ & 82 & 39.5 & 39.3 & $0.5 \%$ \\
\hline 33 & 40.74 & 39.9 & $2.0 \%$ & 83 & 39.5 & 39.5 & $0.1 \%$ \\
\hline 34 & 40.74 & 39.3 & $3.4 \%$ & 84 & 39.5 & 39.2 & $0.7 \%$ \\
\hline 35 & 40.74 & 38.6 & $5.3 \%$ & 85 & 39.5 & 39.2 & $0.7 \%$ \\
\hline 36 & 17.0 & 18.0 & $5.7 \%$ & 86 & 16.5 & 17.2 & $4.5 \%$ \\
\hline 37 & 17.0 & 17.8 & $4.9 \%$ & 87 & 16.5 & 17.0 & $3.3 \%$ \\
\hline 38 & 17.0 & 18.2 & $6.8 \%$ & 88 & 16.5 & 17.1 & $3.7 \%$ \\
\hline 39 & 17.0 & 18.3 & $7.6 \%$ & 89 & 16.5 & 17.1 & $3.4 \%$ \\
\hline 40 & 17.0 & 18.3 & $7.9 \%$ & 90 & 16.5 & 16.8 & $1.8 \%$ \\
\hline 41 & 20.4 & 20.9 & $2.3 \%$ & 91 & 19.7 & 20.2 & $2.8 \%$ \\
\hline 42 & 20.4 & 21.0 & $3.0 \%$ & 92 & 19.7 & 20.0 & $1.5 \%$ \\
\hline 43 & 20.4 & 21.2 & $3.8 \%$ & 93 & 19.7 & 20.3 & $2.9 \%$ \\
\hline 44 & 20.4 & 21.1 & $3.7 \%$ & 94 & 19.7 & 20.0 & $1.6 \%$ \\
\hline 45 & 20.4 & 21.1 & $3.7 \%$ & 95 & 19.7 & 20.2 & $2.7 \%$ \\
\hline 46 & 23.8 & 24.2 & $1.5 \%$ & 96 & 23.0 & 22.7 & $1.4 \%$ \\
\hline 47 & 23.8 & 24.1 & $1.3 \%$ & 97 & 23.0 & 22.6 & $1.6 \%$ \\
\hline 48 & 23.8 & 24.1 & $1.3 \%$ & 98 & 23.0 & 22.7 & $1.4 \%$ \\
\hline 49 & 23.8 & 24.2 & $1.6 \%$ & 99 & 23.0 & 22.7 & $1.4 \%$ \\
\hline 50 & 23.8 & 24.0 & $0.9 \%$ & 100 & 23.0 & 22.9 & $0.6 \%$ \\
\hline
\end{tabular}




\begin{tabular}{|c|c|c|c|c|c|c|c|}
\hline Sample & $\begin{array}{l}\text { Actual Concentration } \\
\qquad(\mathrm{mg} / \mathrm{mL})\end{array}$ & $\begin{array}{l}\text { Predicted Concentration } \\
\qquad(\mathrm{mg} / \mathrm{mL})\end{array}$ & Relative Error (\%) & Sample & $\begin{array}{l}\text { Actual Concentration } \\
\qquad(\mathrm{mg} / \mathrm{mL})\end{array}$ & $\begin{array}{l}\text { Predicted Concentration } \\
\qquad(\mathrm{mg} / \mathrm{mL})\end{array}$ & Relative Error (\%) \\
\hline 101 & 26.3 & 30.0 & $14.2 \%$ & 151 & 34.5 & 34.5 & $0.0 \%$ \\
\hline 102 & 26.3 & 30.0 & $14.2 \%$ & 152 & 34.5 & 34.4 & $0.3 \%$ \\
\hline 103 & 26.3 & 30.1 & $14.3 \%$ & 153 & 34.5 & 34.5 & $0.1 \%$ \\
\hline 104 & 26.3 & 30.3 & $15.4 \%$ & 154 & 34.5 & 34.2 & $1.0 \%$ \\
\hline 105 & 26.3 & 30.1 & $14.5 \%$ & 155 & 34.5 & 34.0 & $1.5 \%$ \\
\hline 106 & 29.6 & 25.9 & $12.5 \%$ & 156 & 32.6 & 32.4 & $0.7 \%$ \\
\hline 107 & 29.6 & 25.8 & $12.9 \%$ & 157 & 32.6 & 32.3 & $1.0 \%$ \\
\hline 108 & 29.6 & 26.0 & $12.0 \%$ & 158 & 32.6 & 32.4 & $0.5 \%$ \\
\hline 109 & 29.6 & 25.9 & $12.5 \%$ & 159 & 32.6 & 32.2 & $1.2 \%$ \\
\hline 110 & 29.6 & 25.9 & $12.6 \%$ & 160 & 32.6 & 32.0 & $1.9 \%$ \\
\hline 111 & 31.3 & 29.0 & $7.2 \%$ & 161 & 20.1 & 20.4 & $1.4 \%$ \\
\hline 112 & 31.3 & 28.9 & $7.6 \%$ & 162 & 20.1 & 20.4 & $1.4 \%$ \\
\hline 113 & 31.3 & 28.7 & $8.4 \%$ & 163 & 20.1 & 20.1 & $0.2 \%$ \\
\hline 114 & 31.3 & 28.8 & $8.0 \%$ & 164 & 20.1 & 20.7 & $2.9 \%$ \\
\hline 115 & 31.3 & 29.0 & $7.5 \%$ & 165 & 20.1 & 19.9 & $0.8 \%$ \\
\hline 116 & 32.9 & 32.4 & $1.6 \%$ & & & & \\
\hline 117 & 32.9 & 32.4 & $1.6 \%$ & & & & \\
\hline 118 & 32.9 & 32.8 & $0.4 \%$ & & & & \\
\hline 119 & 32.9 & 32.7 & $0.6 \%$ & & & & \\
\hline 120 & 32.9 & 32.5 & $1.2 \%$ & & & & \\
\hline 121 & 34.5 & 34.2 & $0.8 \%$ & & & & \\
\hline 122 & 34.5 & 34.8 & $0.8 \%$ & & & & \\
\hline 123 & 34.5 & 34.6 & $0.4 \%$ & & & & \\
\hline 124 & 34.5 & 33.9 & $1.7 \%$ & & & & \\
\hline 125 & 34.5 & 34.6 & $0.4 \%$ & & & & \\
\hline 126 & 36.1 & 37.2 & $3.1 \%$ & & & & \\
\hline 127 & 36.1 & 37.0 & $2.5 \%$ & & & & \\
\hline 128 & 36.1 & 37.0 & $2.6 \%$ & & & & \\
\hline 129 & 36.1 & 36.9 & $2.2 \%$ & & & & \\
\hline 130 & 36.1 & 37.2 & $3.0 \%$ & & & & \\
\hline 131 & 39.5 & 40.1 & $1.6 \%$ & & & & \\
\hline 132 & 39.5 & 40.0 & $1.2 \%$ & & & & \\
\hline 133 & 39.5 & 39.8 & $0.9 \%$ & & & & \\
\hline 134 & 39.5 & 39.7 & $0.6 \%$ & & & & \\
\hline 135 & 39.5 & 40.1 & $1.6 \%$ & & & & \\
\hline 136 & 33.8 & 34.7 & $2.6 \%$ & & & & \\
\hline 137 & 33.8 & 35.2 & $4.0 \%$ & & & & \\
\hline 138 & 33.8 & 34.7 & $2.8 \%$ & & & & \\
\hline 139 & 33.8 & 35.0 & $3.5 \%$ & & & & \\
\hline 140 & 33.8 & 34.9 & $3.3 \%$ & & & & \\
\hline 141 & 34.4 & 33.9 & $1.6 \%$ & & & & \\
\hline 142 & 34.4 & 34.1 & $1.0 \%$ & & & & \\
\hline 143 & 34.4 & 33.8 & $1.8 \%$ & & & & \\
\hline 144 & 34.4 & 34.2 & $0.5 \%$ & & & & \\
\hline 145 & 34.4 & 34.0 & $1.1 \%$ & & & & \\
\hline 146 & 34.5 & 33.9 & $1.8 \%$ & & & & \\
\hline 147 & 34.5 & 34.1 & $1.3 \%$ & & & & \\
\hline 148 & 34.5 & 33.8 & $2.1 \%$ & & & & \\
\hline 149 & 34.5 & 34.2 & $0.8 \%$ & & & & \\
\hline 150 & 34.5 & 34.0 & $1.3 \%$ & & & & \\
\hline
\end{tabular}

Table S7. A comparison of Step 2 FlowIR models built using different modeling approaches for predicting the cyclopropyl-enamine 5 concentration. Model A with four latent variables, wavenumber range from 704 to $1764 \mathrm{~cm}^{-1}$, and preprocessing with Savitzky-Golay $2^{\text {nd }}$-order derivative was selected for this study based on RMSEC, RMSECV, and $\mathrm{r}^{2}$ values. 


\begin{tabular}{|c|c|c|c|c|c|c|}
\hline Model ID & $\begin{array}{l}\text { RMSEC } \\
(\mathrm{mg} / \mathrm{mL})\end{array}$ & $\begin{array}{l}\text { RMSECV } \\
(\mathrm{mg} / \mathrm{mL})\end{array}$ & $r^{2} \mathrm{CV}$ & Latent Variables & Preprocessing & $\begin{array}{c}\text { Wavenumber Range } \\
\left(\mathrm{cm}^{-1}\right)\end{array}$ \\
\hline$\underset{\text { (selected) }}{\mathrm{A}}$ & 2.71 & 2.79 & 0.987 & 4 & \multirow{3}{*}{$\begin{array}{l}\text { Savitzky-Golay } 2^{\text {nd }} \text {-order } \\
\text { polynomial with } 15 \mathrm{pt} \\
\text { smoothing }\end{array}$} & \multirow{4}{*}{ 704-1764 } \\
\hline B & 3.36 & 3.50 & 0.980 & 2 & & \\
\hline $\mathrm{C}$ & 2.86 & 2.94 & 0.986 & 3 & & \\
\hline D & 3.76 & 3.92 & 0.974 & 3 & None & \\
\hline
\end{tabular}

Table S8. A comparison of Step 5 Raman models built using different modeling approaches for predicting the ciprofloxacin 8 concentration. The spectral data were mean centered and preprocessed with AWLS baseline and EMSC filters. Model A with 7 latent variables with spectra regions from 768 to $971 \mathrm{~cm}^{-1}$ and from 1077 to $1660 \mathrm{~cm}^{-1}$ was selected.

\begin{tabular}{|c|c|c|c|c|c|c|}
\hline Model ID & $\begin{array}{l}\text { RMSEC } \\
(\mathrm{mg} / \mathrm{mL})\end{array}$ & $\begin{array}{c}\text { RMSECV } \\
\text { (mg/mL) }\end{array}$ & $r^{2} \mathrm{CV}$ & Latent Variables & Preprocessing & $\begin{array}{l}\text { Wavenumber Range } \\
\left(\mathrm{cm}^{-1}\right)\end{array}$ \\
\hline $\begin{array}{c}\mathrm{A} \\
\text { (selected) }\end{array}$ & 1.12 & 1.29 & 0.969 & 7 & \multirow{4}{*}{$\begin{array}{l}\text { *AWLS Baseline, } \\
\text { EMSC, Mean Centered }\end{array}$} & $768-971,1077-1660$ \\
\hline B & 2.24 & 2.26 & 0.964 & 2 & & 283-1904 \\
\hline $\mathrm{C}$ & 2.15 & 2.18 & 0.966 & 3 & & $768-971,1077-1660$ \\
\hline $\mathrm{D}$ & 1.84 & 1.86 & 0.935 & 2 & & $768-971,1077-1660$ \\
\hline
\end{tabular}

\section{PAT Data Collection and Management}

synTQ was utilized for PAT data collection and management of various hardware and software needed for PAT implementation on the PoD synthesis unit. The software for FlowIR (iC IR version 4.3) and the software for online PLS model processing (Solo_Predictor) were installed on the synTQ computer and configured using the synTQ's built-in adaptor functionality. These adaptors were used to establish communications between synTQ and instruments as well as iC IR and Solo_Predictor. The MarqMetrix All-in-One Raman spectrometer was controlled independently and configured with synTQ via ethernet for real-time data transfer and processing. For this purpose, a secure local network was established within the laboratory. In synTQ, the spectral data collected from both FlowIR and Raman spectroscopy were processed by Solo_Predictor for product concentration predictions using the corresponding PLS models. Figure S7 shows an example of synTQ during a synthesis run and Figure S8 is an example of the operator view for concentration predictions of Steps 2 and 5. 


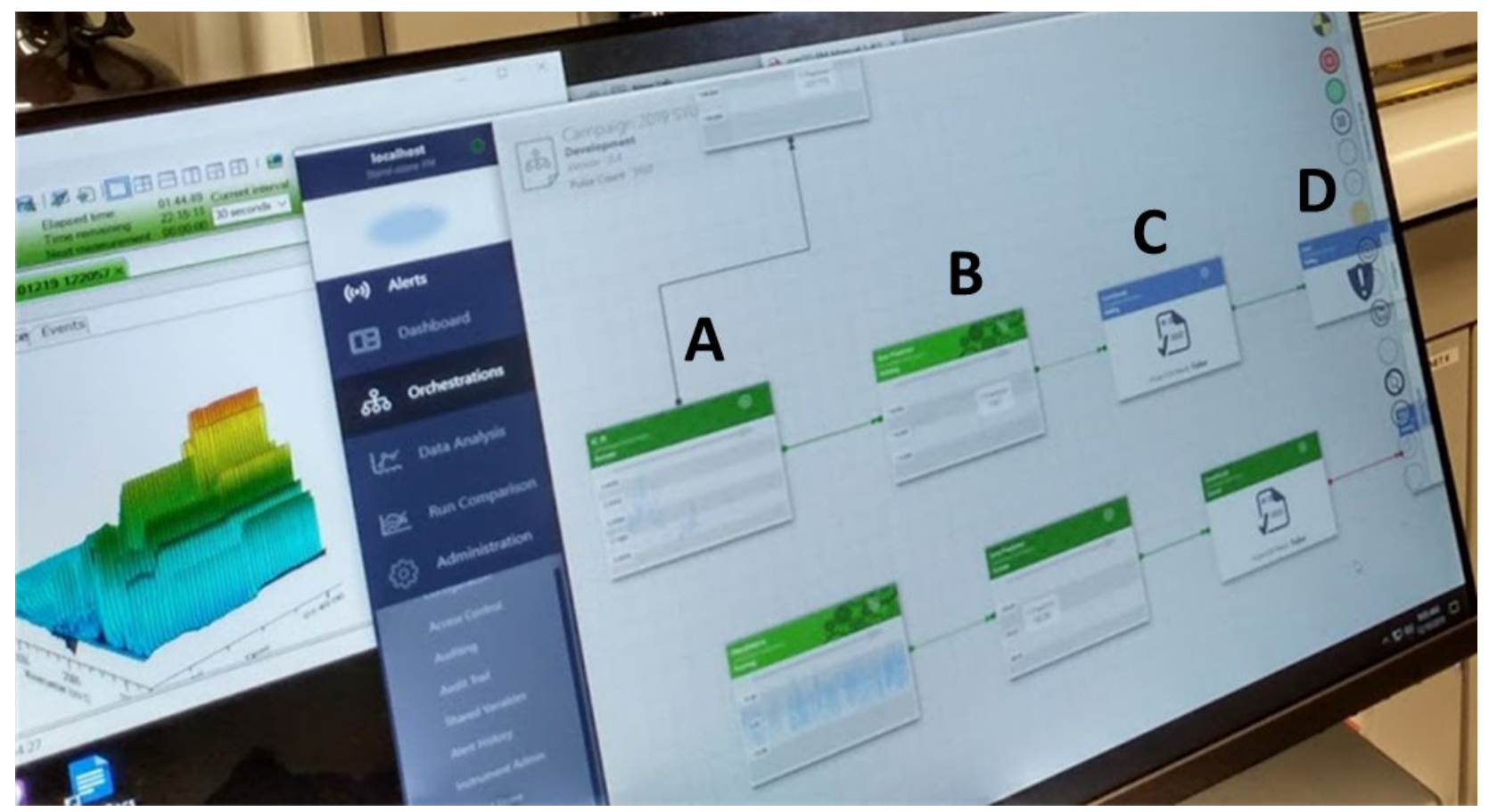

Figure S7. An example of PAT data collection and management with synTQ: (A) continuous spectral data from FlowIR via iC IR are collected and stored in synTQ, (B) real-time concentration prediction with Solo_Predictor is achieved using Step 2 FlowIR model, (C) a logic statement for monitoring Step 2 which triggers an alert system, and (D) operators will be alerted when product concentration is outside of the target concentration range. 

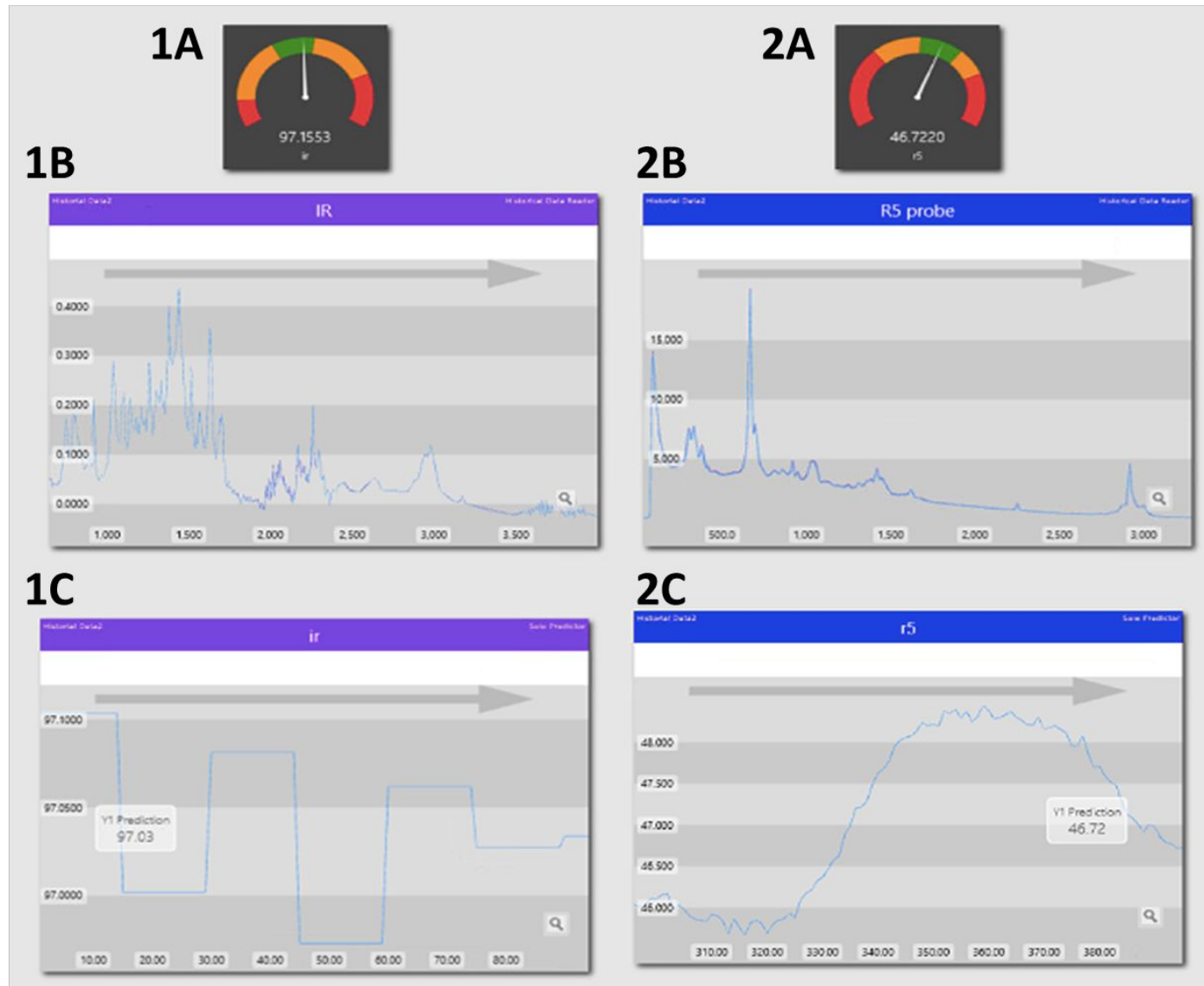

Figure S8. An example of synTQ real time monitoring functionalities with instantaneous concentration predictions of cyclopropyl-enamine 5 (1A) and ciprofloxacin 8 (2A) determined from raw IR (1B) and Raman (2B) spectra. The concentration predictions of cyclopropyl-enamine 5 (1C) and ciprofloxacin 8 (2C) over a period of time are shown.

\section{Reference}

(1) Armstrong, C.; Miyai, Y.; Formosa, A.; Thomas, D.; Chen, E.; Hart, T.; Schultz, V.; Desai, B. K.; Cai, A. Y.; Almasy, A.; Jensen, K.; Rogers, L.; Roper, T. On-Demand Continuous Manufacturing of Ciprofloxacin in Portable Plug-and-Play Factories: Development of a Highly Efficient Synthesis for Ciprofloxacin. Org. Process Res. Dev. 2021. https://doi.org/10.1021/acs.oprd.1c00118. 